\title{
Improving Profitability of Optimal Mean Setting with Multiple Feature Means for Dual Quality Characteristics
}

\author{
C. Dodd • J. Scanlan • R. Marsh • S. Wiseall
}

Received: date / Accepted: date

\begin{abstract}
The setting of a process mean for a manufacturing process which frequently produces scrap and rework, can significantly affect profitability. Optimal mean setting is a methodology by which the process mean is adjusted to maximise profit. This paper studies the dynamics of the problem and investigates the possibility of applying different process means to each rework iteration, to further maximise profit. A proof is given confirming there is only one optimal mean that applies over all rework iterations in the single feature case. However, applying similar reasoning to a dual feature case led to the development of a new optimal mean setting methodology which outperformed the existing approach in terms of the maximum expected profit.
\end{abstract}

Keywords Optimum process mean - Dual quality characteristics · Optimisation · Quality control

\section{Introduction}

Optimal mean setting is the practice of adjusting manufacturing parameters and machine settings to control the location of the process mean to maximise profit. The principle has a long standing history originating as the 'canning problem' posed by Springer in 1951

C. Dodd · J. Scanlan · R. Marsh

Computational Engineering \& Design Group

Engineering Centre of Excellence

Boldrewood Campus

University of Southampton, SO17 1BJ

Tel.: +44 (0) 7976099985, Fax.: +44 (0) 2380594813

E-mail: cd205@soton.ac.uk, E-mail: J.P.Scanlan@soton.ac.uk

S. Wiseall

Product Cost Engineering

Rolls-Royce PLC, Moor Lane

Derby, DE24 8BJ
[1]. The motivation for the practice stems from the differences between scrap and rework costs and generally applies to manufacturing processes where the common cause variation of the process is greater than the feature specification limits (tolerance bands). A normal distribution is often used to model the manufacturing process variation, as illustrated in Figure 1. Rework is created when a manufacturing operation produces a feature (or quality characteristic) outside the specification limits (non-conforming), where additional manufacturing operations can bring that feature inside the specification limits. For material removal type operations, rework is typically generated when the inspected feature is larger than the upper specification limit (U), illustrated by the striped region in Figure 1. Scrap is created when a feature is non-conforming and no additional manufacturing operations can make that feature conform. For material removal operations this typically implies the feature is under the lower specification limit (L), illustrated by the cross hatched region in Figure 1 .

The cost of scrapping components is generally higher than the costs of reworking thus, in Figure 1, the sum of scrap and rework cost would be reduced by shifting the mean $(\mu)$ to the right. This would increase the proportion of features requiring rework while reducing the proportion of features that would lead to scrap. The fundamental requirement of optimal mean setting is to maximise profit (rather than minimise scrap and rework cost) by maximising an expression in the form of Equation 1. The number of items sold, scrap cost and rework cost are functions of the process mean, $\mu$. The processing cost is the cost of the initial manufacturing operation and is generally considered to be constant.

$\begin{aligned} \text { Profit } & =\text { Items Sold }- \text { Processing Cost } \\ & - \text { Scrap Cost }- \text { Rework Cost } .\end{aligned}$ 


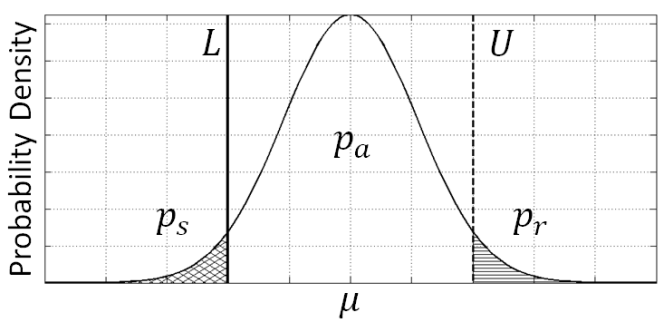

Fig. 1 Illustration of the rework $\left(p_{r}\right)$, conformance $\left(p_{a}\right)$ and scrap $\left(p_{s}\right)$ probabilities

It is possible for several features to be manufactured, either by serial or parallel manufacturing operations. In a serial manufacturing system, one feature is manufactured and then inspected, before the next feature is manufactured and inspected. In a parallel manufacturing system two or more features are manufactured before being inspected. Dual feature (or dual quality characteristic) manufacturing is parallel manufacturing with exactly two features. In both serial and parallel cases it is possible for a feature to be reworked multiple times before being deemed scrap or conforming. Furthermore, for parallel production, different types of rework are possible as only one feature or multiple features may require rework. In such cases different mean values may be applied to each rework iteration or type of rework. This article establishes how such an approach can increase in the maximum expected profit obtainable through optimal mean setting compared to the existing literature.

\section{Literature Review}

Several researches [2-5] extended the original work of Springer's can filling problem [1], where the optimal filling level of a can was sought by adjusting the specification limits. One of the assumptions in these models was the existence of a secondary market, where nonconforming product could be sold at a discounted rate. Bettes [6] proposed an alternative model for products such as pharmaceuticals, where no secondary market existed and all products had to conform. The upper limit was optimised to reduce the loss incurred when customers received extra product but at the standard price. The concept was advanced by Golhar [7], Schmidt and Pfeifer [8] and Liu and Raghavachari [9]. Wen and Mergen [10] were the first to apply optimal mean setting to a feature manufacturing problem. The mean for a grinding operation was optimised for the production of an inner ring of a bearing race. Several researchers ([11-14] introduced the Taguchi loss function [15] to the
Wen and Mergen problem, this addition limited the extent to which the mean was biased towards rework.

A number of researchers [16-19] investigated optimal mean setting of dual features (parallel manufacturing), where the quality loss was modelled using the bivariate normal distribution function. Chen and Chou [14] extended the work by considering different nonconformance costs depending if a feature was greater or lower than the upper and lower specification limits, respectively. Al-Sultan and Pulak [20] were the first to consider multiple feature in series, which was an extension of [21] but for two manufacturing stages. Bowling et al. [22] and Khasawneh et al. [23] introduced Markovian modelling to the optimal mean setting problem for serial and parallel process, respectively. Prior to this, rework was considered as a static one-off cost, however, rework is dynamic and several rework operations maybe required before features either conform or are scrapped. The assumption that a feature will conform following a single rework operation underestimates the total rework required and scrap produced.

Selim and Al-Zu'bi [24] further refined the Markovian model presented by Bowling et al. [22] and corrected an error in the model for multiple features manufactured in series. Peng and Khasawneh [25] modified the dual feature approach proposed by Khasawneh et al. [23] and applied it to a production system where a sampling plan was used to inspect feature quality, rather than a $100 \%$ inspection. The effect of correlation between features was studied as well as a two stage production system where dual features were produced at each stage.

Goethals and Cho [26] derived the most cost effective process mean and variation through observation and design of experiment. This is in contrast to the literature discussed up to this point, where the variance of a process was assumed to be fixed. A response surface for the process mean and variance was modelled in response to several process variables and optimised to minimise total cost. Goethals and Cho [27] and Boylan and Cho [28] extended the problem for multiple quality characteristics and also employed the skew normal distribution [29] to represent different quality characteristics. This approach was not taken in the article presented here as the mean and variance of manufacturing processes are assumed to be known.

The Markovian approach to optimal mean setting is a truer representation of a manufacturing system where rework is produced $[23,24]$. In such a system it is feasible to adjust the process mean for each operation or set different target means for different types of reworks in an effort to further increase profit (for parallel manufacturing). This has not been considered in the literature. 


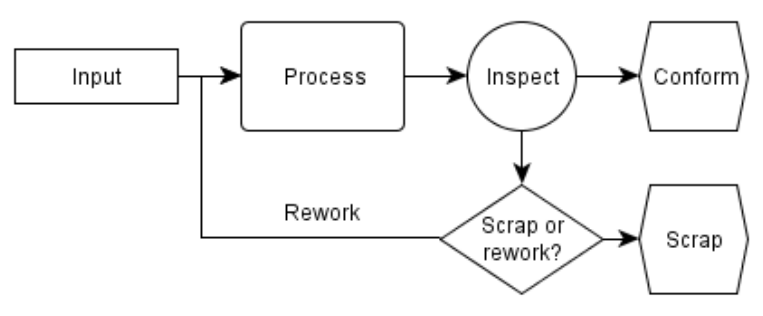

Fig. 2 Process flow diagram with a rework loop

In this article, the equations for expected profit are derived to allow different mean settings for each rework operation. The production of a single feature in series, with multiple rework means is considered in Section 3. Section 4, develops a dual feature model with multiple rework means specifically allowing the means of the different rework types to be optimised separately. An increase in the maximum profit is shown to be achievable compared to the methodology outlined by Khasawneh et al. [23] and used by Peng and Khasawneh [25]. The effect correlation between features has on the maximum profit is also considered for both the Khasawneh's method and the new implementation.

\section{Optimal Mean Setting - Single Feature}

A manufacturing method involving rework is an iterative process starting with an initial cut which generates features in three states; rework, conforming and scrap. This is illustrated by Figure 2 where an inspection operation is used to determine which states the features belong to. Subsequent operations convert the rework into just two states, conforming and scrap. For example, after manufacturing a batch of components a certain number of features may be designated rework. This initial manufacturing operation is referred to as the first iteration. The processing of this rework is referred to as the second iteration. After the second iteration it is possible some items will still require rework, the reprocessing of this rework is referred to as the third iteration. The process continues until all rework items are either conform or are scrapped. The probability of features conforming, being scrapped or requiring rework is determined by evaluating the areas under the probability density function illustrated for two iterations in Figure 3. The left plot shows the initial manufacturing operation, which was positioned with an off-centre mean, $\mu_{x_{1}}=6.5$, to minimise scrap in favour of rework. The standard deviation was set at $\sigma=1$. The striped area represents the features requiring rework while the white area under the curve represents conforming features, there was no appreciable scrap. The second it- eration (right plot in Figure 3) indicates the result of reprocessing the rework features. The grey dashed line shows the resulting distribution from this second iteration. The solid black line in Figure 3 is the sum of the conforming components from the first and second iterations and represents the distribution of manufactured geometry after the second iteration. The smaller striped area indicates further rework is required. Subsequent iterations would steadily reduce this rework until all components were either conforming or scrap.

To maximise profit from such a process, an optimal reworking strategy must be identified, specifically the mean values for each iteration. It would be feasible to alter the mean values for each iteration such that the initial mean may be $\mu_{x_{1}}=6.5$, followed by a different mean for subsequent iterations. However, it can be shown for the production of a single feature, only one optimal mean exists (to maximise profit) for the initial manufacturing stage and all subsequent rework operations. It is assumed;

- the manufacturing variation of the initial operation and all rework operations are the same, i.e. the same or a similar machine is used.

- the specification limits for the initial operation and all rework operations are the same, i.e. the nominal geometry of the feature is unchanged.

The expected profit can be expressed as,

$$
\begin{aligned}
& E(P R)=S P\left[\sum_{i=1}^{n}\left[F\left(U, \mu_{i}, \sigma\right)-F\left(L, \mu_{i}, \sigma\right)\right] \prod_{i=2}^{n}\right. \\
& \left.\left[1-F\left(U, \mu_{i-1}, \sigma\right)\right]\right]-P C-\left[\sum _ { i = 1 } ^ { n } \left(S c\left[F\left(L, \mu_{i}, \sigma\right)\right]\right.\right. \\
& \left.\left.+R c\left[1-F\left(U, \mu_{i}, \sigma\right)\right]\right) \prod_{i=2}^{n}\left[1-F\left(U, \mu_{i-1}, \sigma\right)\right]\right]
\end{aligned}
$$

which corresponds to Equation 1 but in mathematical form. The constants, $S P, P C, S c$, and $R c$ are the selling price, processing cost, scrap cost and rework cost respectively. The means, $\mu_{i}$ for $i=1,2, \ldots, \infty$, are the target means for each iteration and the standard deviation is given by $\sigma$. The initial operation is $i=1$ and $i \geq 2$ are rework iterations. For $i=1$, the first term in the square parentheses relates to the white area under the 'Initial distribution' in the first plot of Figure 3. The second set of parentheses relates to the area of the rework (striped region) and scrap regions (first plot in Figure 3). The scrap area, given by $F\left(L, \mu_{1}, \sigma\right)$ is close to zero in Figure 3. When $i=2$ the product terms become relevant, accounting for the number of 

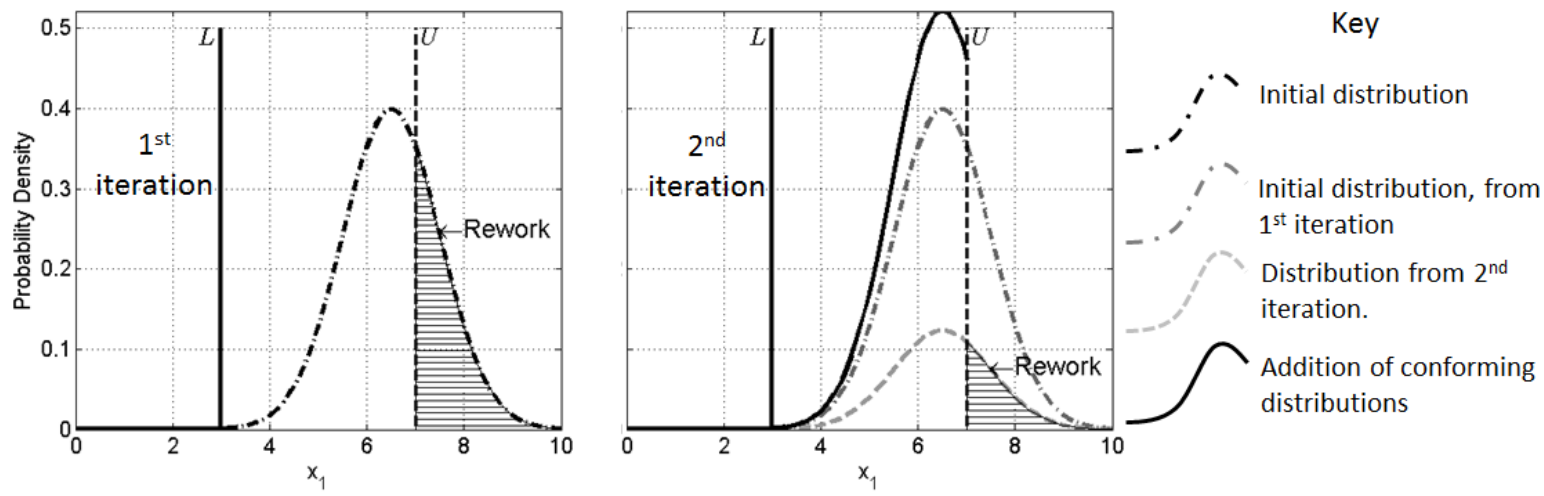

Fig. 3 Two iterations of a process with rework

items that were designated rework from the previous iteration. Thus, the first term in the square parentheses (Equation 2) relates to the white area under the 'Distribution from 2nd iteration', (second plot of Figure 3). Similarly the second set of parenthesis in Equation 2 relates to the rework and scrap regions under the 'Distribution from 2nd iteration' curve in Figure 3. For practical situations $n$ is a large number defining the total number of iterations necessary to complete all the rework such that only scrap and conforming items remain. The function $F(\bullet)$ is the cumulative normal distribution function (CDF) given by,

$F(X, \mu, \sigma)=\operatorname{Pr}[X \leq x]=\int_{-\infty}^{x} f_{X} \mathrm{~d} t$

where $f_{X}$ is the normal distribution function given by,

$f(x, \mu, \sigma)=\frac{1}{\sigma \sqrt{2 \pi}} \exp \left(-\frac{(x-\mu)^{2}}{2 \sigma^{2}}\right)$.

Equation 2 determines the difference between the income generated from the components that can be sold (first term) and the production cost. The production cost includes the costs of scrap and rework, enclosed within the second set of large square parenthesis, and the initial processing cost $(P C)$.

It is conjectured that to maximise the profit an optimal mean can be found which is the same for every iteration (the initial processing and all rework iterations). This is proven here.

Theorem 1 There is only one $\mu_{\text {opt }}$ that satisfies

$$
\max _{\boldsymbol{\mu} \in \mathbb{R}}\{T C(\boldsymbol{\mu})\},
$$

where $\boldsymbol{\mu}=\left[\mu_{1}, \mu_{2}, \ldots, \mu_{\infty}\right]$. Such that $\mu_{i}=\mu_{o p t}, \forall i \exists[1, \infty]$

Proof Setting Equation 2 to zero and differentiating with respect to each $\mu_{i}, \partial T P_{i} / \partial \mu_{i}$ gives the stationary point $(\text { maximum })^{1}$ for each iteration $i$. A general expression for the maximum for each iteration is sought. Although $i \rightarrow \infty$, in general the number of rework iterations for a batch of components will be finite. However, there is always a diminishingly small probability that rework will exist and more iterations will be required. Let the total number of rework iterations be $n$ where in practical cases $n$ will be a large number but in the general case $n=\infty$. Consider the optimal means for the last three rework iterations,

$\mu_{\mathrm{opt}_{n}}=\frac{1}{2(L-U)}\left\{2 \sigma^{2} \ln \left[\frac{S P+R c}{S P+S c}\right]+L^{2}-U^{2}\right\}$

$$
\begin{aligned}
\mu_{\mathrm{opt}_{n-1}} & =\frac{1}{2(L-U)}\left\{2 \sigma ^ { 2 } \operatorname { l n } \left[\frac { 1 } { 2 \alpha } \left(\xi\left(\varphi_{n}\right) \alpha\right.\right.\right. \\
& \left.\left.-\xi\left(v_{n}\right) \beta+3 R c+2 S P+S c\right)\right] \\
& \left.+L^{2}-U^{2}\right\}, \\
\mu_{\mathrm{opt}_{n-2}} & =\frac{1}{2(L-U)}\left\{2 \sigma ^ { 2 } \operatorname { l n } \left[\frac { 1 } { 4 \alpha } \left(\left(-\xi\left(\varphi_{n}\right) \alpha\right.\right.\right.\right. \\
& \left.-\xi\left(v_{n}\right) \beta-3 R c-2 S P-S c\right) \xi\left(v_{n-1}\right) \\
& +\xi\left(\varphi_{n}\right) \alpha+\xi\left(v_{n}\right) \beta-2 \xi\left(\varphi_{n-1}\right) \alpha \\
& \left.+7 R c+4 S P+3 S c)]+L^{2}-U^{2}\right\} .
\end{aligned}
$$

The stationary point is shown to be a maximum after the proof is completed, rather than showing each stationary point is a maximum for every $i$. This is shown in the Appendix, Section 6.1. 
There are two cost terms defined as $\alpha=S P+S c$ and $\beta=S P+R c$ and $\xi$ is the error function given by,

$\xi\left(\varphi_{i}\right)=\frac{2}{\sqrt{\pi}} \int_{0}^{\varphi_{i}} e^{-t} \mathrm{~d} t$ and $\xi\left(v_{i}\right)=\frac{2}{\sqrt{\pi}} \int_{0}^{v_{i}} e^{-t} \mathrm{~d} t$

where,

$\varphi_{i}=\frac{\sqrt{2}\left(-\mu_{i}+L\right)}{2 \sigma}$ and $v_{i}=\frac{\sqrt{2}\left(-\mu_{i}+U\right)}{2 \sigma}$

For $i=n, \mu_{\mathrm{opt}_{n}}$ is purely a function of the relative costs and relationship between the specification limits ( $L$ and $U$ ) and the manufacturing variation $\sigma$. The second to last optimal mean, $\mu_{\mathrm{opt}_{n-1}}$, is a function of the costs, specification limits, the standard deviation and the last optimal mean, $\mu_{\mathrm{opt}_{n}}$. The third to last optimal mean, $\mu_{\mathrm{opt}_{n-2}}$, is a function of the costs, specification limits, standard deviation and the last two optimal means, $\mu_{\mathrm{opt}_{n-1}}$ and $\mu_{\mathrm{opt}_{n}}$. Notice that the earlier optimal means are functions of all subsequent optimal means; thus to establish the value of the first optimal mean, one must first establish the value of the last optimal mean, then the second to last optimal mean and so on. Accordingly, a new subscript $j$ is defined such that $j=[n, n-1, \ldots, 1]$. From Equations 5 to 7 a general expression for $\mu_{\mathrm{opt}_{j}}$ can be constructed where,

$\mu_{\mathrm{opt}_{j}}=\frac{1}{2(L-U)}\left\{2 \sigma^{2} \ln \left[\frac{\Gamma_{j}}{2^{n-j} \alpha}\right]+L^{2}-U^{2}\right\}$

and $\Gamma_{j}$ is given by,

$$
\begin{aligned}
\Gamma_{j}= & -1\left[\Gamma_{j+1} \xi\left(v_{j+1}\right)\right] \\
& +\Gamma_{j+1}+2^{n-(j+1)} \xi\left(\varphi_{j+1}\right) \alpha \\
& +2^{n-j} R c+2^{n-(j+1)} S P+2^{n-(j+1)} S c .
\end{aligned}
$$

The $n^{\text {th }}$ term is always

$\mu_{\mathrm{opt}_{n}}=\frac{1}{2(L-U)}\left\{2 \sigma^{2} \ln \left[\frac{\beta}{\alpha}\right]+L^{2}-U^{2}\right\}$.

Lemma 1 Given that $L, U$ and $\sigma$ remain constant for each iteration, to prove the conjecture, $\mu_{i}=\mu_{o p t}$, $\forall i \exists[1, \infty]$, it must be shown that,

$$
\begin{aligned}
\frac{\Gamma_{j}}{2^{n-j} \alpha} & =\left.\frac{\Gamma_{j+1}}{2^{n-j+1} \alpha}\right|_{n \rightarrow \infty} \text { which reduces to } \\
\Gamma_{j} & =\left.2 \Gamma_{j+1}\right|_{n \rightarrow \infty}
\end{aligned}
$$

as the denominator for the $j+1$ term is double the $j^{\text {th }}$ term. The last three terms of $\Gamma$, Equation 11, increase as a factor of two for each iteration. The third term, $2^{n-(j+1)} \xi\left(\varphi_{j+1}\right) \alpha$, can increase up to a maximum of a factor of two for each iteration, when $\varphi_{j+1}=1$. Thus, it remains to be shown the maximum rate of increase, per iteration, for the first two terms of $\Gamma$ is two, in the limit $n \rightarrow \infty$. This is shown by implementing linear stability analysis. A new subscript $m$ is defined where $m=[n-1, n-2, \ldots, 1]$ where $m$ is the next point after $m+1$. Let $f(\Gamma)=\Gamma_{m} / 2^{n-m} \alpha$ and let a fixed point be defined such that

$\Gamma_{m}=\Gamma_{m+1}=\Gamma^{*}=f\left(\Gamma^{*}\right)$.

A small deviation from this fixed point is,

$$
\Gamma_{m+1}=\Gamma^{*}+\delta \Gamma_{m+1}
$$

Therefore at the next step

$$
\begin{aligned}
\delta \Gamma_{m} & =\Gamma_{m}-\Gamma^{*} \\
& =f\left(\Gamma_{m+1}\right)-\Gamma^{*} \\
& =f\left(\Gamma^{*}+\delta \Gamma_{m+1}\right)-\Gamma^{*}
\end{aligned}
$$

Since $\delta \Gamma_{m+1}<<\Gamma^{*}$ a Taylor series expansion around $\Gamma^{*}$ can be implemented giving,

$f\left(\Gamma^{*}+\delta \Gamma_{m+1}\right)=\Gamma^{*}+\delta \Gamma_{m+1}\left(\frac{\mathrm{d} f}{\mathrm{~d} \Gamma}\right)_{\Gamma=\Gamma *}+\mathcal{O}\left(\delta \Gamma_{m+1}^{2}\right)$.

Close to the fixed point the second order terms $\mathcal{O}\left(\delta \Gamma_{m+1}^{2}\right)$ are very small and can be neglected. Recognising $f\left(\Gamma^{*}\right)=$ $\Gamma^{*}$, from Equation 14, the above equation can be rewritten as

$$
\delta \Gamma_{m}=f^{\prime}\left(\Gamma^{*}\right) \delta \Gamma_{m+1}
$$

where $f^{\prime}=\mathrm{d} f / \mathrm{d} \Gamma$ and

$f^{\prime}\left(\Gamma^{*}\right)=-\xi(v)+1$.

The maximum value of Equation 16 is $f^{\prime}\left(\Gamma^{*}\right)=2$ for all values $\mu \exists \mathbb{R}$. Thus, the equality in Equation 13 is satisfied in the limit as $n \rightarrow \infty$ proving Lemma 1 and hence completing the proof, confirming the same optimal mean must be applied for each rework iteration to maximise profit. Thus, the Markovian method (outlined by Bowling et al. [22] and Selim and Al-Zubi [24]), which implicitly uses the same mean for every iteration, is justified.

\subsection{Single Feature Numerical Example}

The solution to a single feature optimal mean setting problem was solved using the proof that the same optimal mean must be applied over all rework iterations to maximise profit (proof in Section 3). The specification limits, process variation, selling price and costs (taken from Bowling et al. [22]) are given by Table 1. 


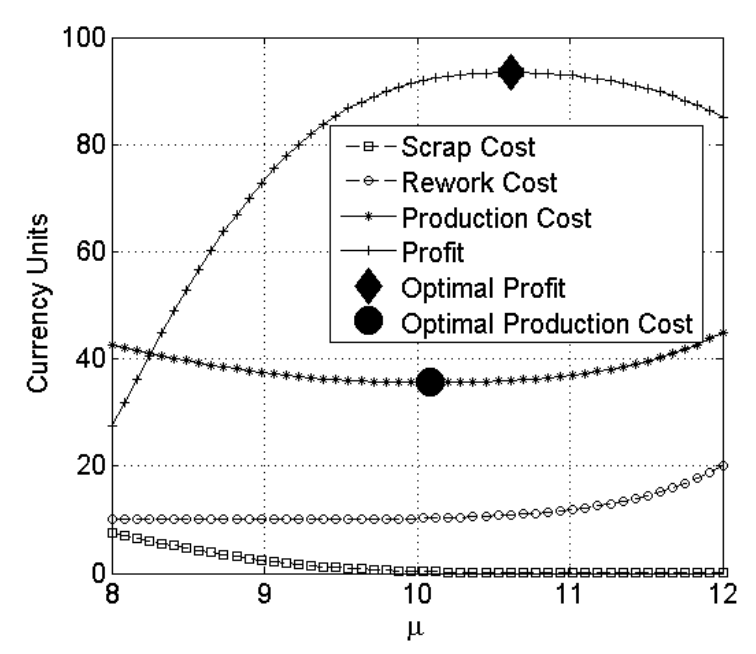

Fig. 4 Profit, scrap, rework, and total production costs

Equation 2 was solved for $8 \leq \mu \leq 12$, the profit, scrap cost, rework cost and production cost are plotted on Figure 4. The results are the same as those produced by the Markovian model from Bowling et al. [22] with a maximum expected profit of 93.4377 at $\mu=10.6144$. This confirms the iterative expression for expected profit (Equation 2) is equivalent to the Markovian model when $\mu_{i}=\mu_{\text {opt }}$. Figure 4 also highlights that the optimal mean for minimum production cost is not the same as the optimal mean to maximise profit at $\mu=10.0875$ and $\mu=10.6144$, respectively. This is due to the increased conformance achieved by more heavily biasing rework and consequently reducing the probability of scrap.

\begin{tabular}{|c|c|c|c|}
\hline Variable & Value & Costs & Value \\
\hline $\mathbf{U}$ & {$\left[\begin{array}{ll}12 & 12\end{array}\right]$} & $\mathrm{SP}$ & 120 \\
\hline $\mathbf{L}$ & {$\left[\begin{array}{ll}8 & 8\end{array}\right]$} & $\mathrm{PC}$ & 25 \\
\hline $\boldsymbol{\sigma}$ & 1 & $\mathrm{SrC}$ & 15 \\
\hline & & $\mathrm{RwC}$ & 10 \\
\hline
\end{tabular}

Table 1 Inputs for the single feature numerical example

\section{Optimal Mean Setting - Dual Features}

A logical extension to optimal mean setting with one feature is a dual feature case, where two features are processed prior to inspection. In the previous section it was shown that maximum profit was attained when the optimal mean for the initial operation and all rework operations were the same. In a dual feature case, three types of rework are produced, where only one state is exactly equivalent to the initial processing operation. Optimal mean setting for dual features was considered

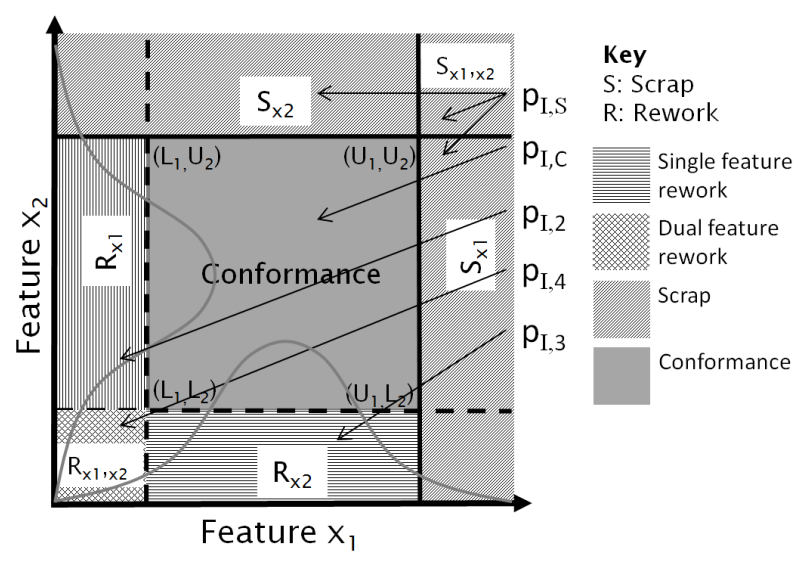

Fig. 6 Dual feature rework, conformance and scrap

by Khasawneh et al. [23] and Peng and Khasawneh [25], however, the Markovian approach used assumed the means remained the same as the initial processing stage irrespective of the rework type. A greater profit is sought here by investigating whether the means for the various rework types should be treated separately.

Figure 5 indicates the processing of two features prior to inspection, reminiscent of the one feature case in Figure 2. Inspection processes are implicit at the end of the initial state and the three rework states. The three rework states are initially fed from the first manufacturing operation (I), which can also cause scrap and conformance. After initial processing, the single feature rework states (2 and 3) may receive components from themselves (i.e. items are reworked but still don't conform and require further rework) or from the dual feature rework state (4) (i.e. only one feature conforms when reworked in state 4 ). The dual feature reworking state (4) can receive components from the initial operation, and also from itself, if after dual feature rework both features still require rework. As in the single feature rework case, all components eventually conform or are scrapped. The initial probabilities of scrap $\left(p_{\mathrm{I}, \mathrm{S}}\right)$, conformance $\left(p_{\mathrm{I}, \mathrm{C}}\right)$ and the three rework states $\left(p_{\mathrm{I}, 2}\right.$, $p_{\mathrm{I}, 3}$ and $\left.p_{\mathrm{I}, 4}\right)$ are illustrated in Figure 6 . These same probabilities apply to state 4 . The axes on Figure 6 have been reversed ( $\infty$ to $-\infty$ rather than $-\infty$ to $\infty$ ) to reduce the number of cumulative distribution function ( $\mathrm{CDF}$ ) evaluations required to find the probability of rework, scrap and conformance. In order to derive an expression to determine the optimal means it is necessary to define expected profit as was done in the one feature example in the Section 3, (Equation 2). Thus, the probabilities of rework, conformance and scrap in the rectangular regions in Figure 6 must be evaluated. Following the principles outlined by Nelson [30], in the general case (for $n$ features) a rectangular region can be defined by; $\boldsymbol{L}=\left(L_{1}, \ldots, L_{n}\right)$ and $\boldsymbol{U}=\left(U_{1}, \ldots, U_{n}\right)$ 


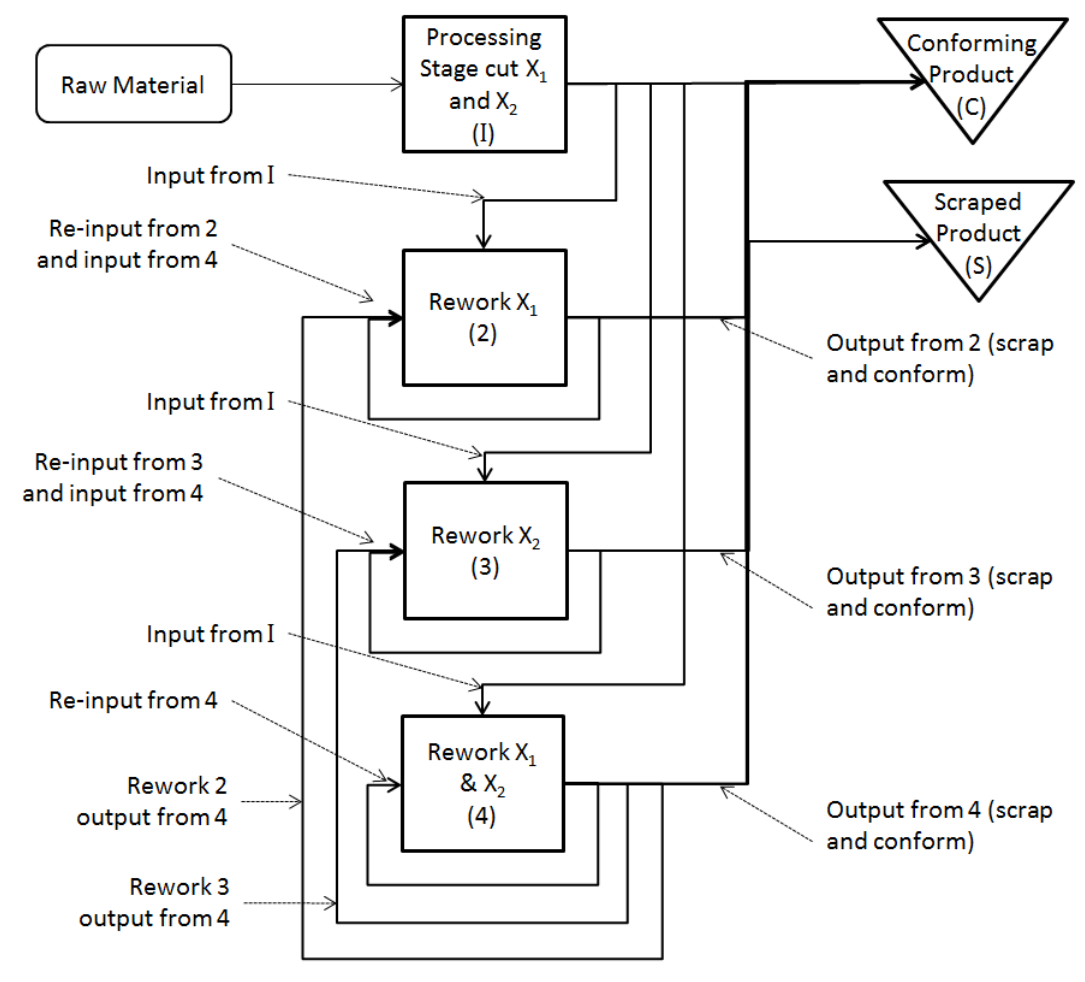

Fig. 5 Manufacturing flow for a two-feature parallel process

where $\boldsymbol{L}_{i} \leq \boldsymbol{U}_{i} \forall i=1,2, \ldots, n$ where $(\boldsymbol{L}, \boldsymbol{U})$ is an ndimensional rectangle and $n$ is the number of features at a given stage. The vectors $\boldsymbol{L}$ and $\boldsymbol{U}$ represent the lower and upper specification limits for each feature as indicated in Figure 6. Taking the Cartesian product of $n$ intervals, $A=\left(L_{1}, U_{1}\right) \times\left(L_{2}, U_{2}\right) \times, \ldots, \times\left(L_{n}, U_{n}\right)$. A cumulative distribution function (CDF) F: $\mathbb{R}^{n} \rightarrow[0,1]$ is given by the integral of the multivariate distribution function,

$F(\boldsymbol{X}, \boldsymbol{\mu}, \boldsymbol{\Sigma})=\int_{-\infty}^{x_{n}} \cdots \int_{-\infty}^{x_{1}} \frac{1}{\sqrt{(2 \pi)^{k}|\boldsymbol{\Sigma}|}}$

$\exp \left\{-\frac{(\boldsymbol{t}-\boldsymbol{\mu})^{T} \boldsymbol{\Sigma}^{-1}(\boldsymbol{t}-\boldsymbol{\mu})}{2}\right\} \mathrm{t}_{1} \ldots \mathrm{t}_{n}$ where,

$$
\left\{\begin{array}{ll}
x_{i_{j}}=L_{j} & \text { if } i_{j}=0, \\
x_{i_{j}}=U_{j} & \text { if } i_{j}=1
\end{array} \quad \forall \quad j=1,2, \ldots, n .\right.
$$

The total probability of rework is simply,

$$
P_{R w}=F(\boldsymbol{U}, \boldsymbol{\mu}, \boldsymbol{\Sigma})-p_{\mathrm{I}, \mathrm{C}}
$$

and the the probability of scrap,

$P_{S r}=1-F(\boldsymbol{U}, \boldsymbol{\mu}, \boldsymbol{\Sigma})$.

where $\boldsymbol{X}$ is a $k$-dimensional random vector $\boldsymbol{X}=\left[X_{1}, \ldots, X_{k}\right]$,

$\boldsymbol{\mu}$ is a $k$-dimensional mean vector $\boldsymbol{\mu}=\left[E\left[X_{1}\right], \ldots, E\left[X_{k}\right]\right]$ The profit equation for two features corresponds to genand $\boldsymbol{\Sigma}$ is a $k \times k$ covariance matrix, $\boldsymbol{\Sigma}=\left[\operatorname{Cov}\left[X_{i}, X_{j}\right]\right], i=$ eral form illustrated by Equation 1 . It follows the same $1, \ldots, k ; j=1, \ldots, k$. The probability of conformance $\left(p_{\mathrm{I}, \mathrm{C}}\right)$ is given by:

$$
p_{\mathrm{I}, \mathrm{C}}=P\left(L_{1}<X_{1} \leq U_{1}, \ldots, L_{n}<X_{n} \leq U_{n}\right)
$$

which can be expressed as,

$p_{\mathrm{I}, \mathrm{C}}=\sum_{i_{1}=0}^{1} \cdots \sum_{i_{n}=0}^{1}(-1)^{i_{1}+\cdots+i_{n}} F\left(x_{i_{1}}, \ldots, x_{i_{n}}\right)$. principles as the one feature profit equation (Equation 2 ), albeit the rework and scrap terms are more complex. As a continuation of the proof, given in Section 3, the means for each iteration are kept constant however, a distinction is made between the dual feature rework means $\boldsymbol{\mu}=\left[\mu_{1,1}, \mu_{1,2}\right]$ and the single feature rework means $\mu_{2,1}$ and $\mu_{2,2}$, for the first and second features respectively (this distinction is justified later in this section). The rework costs for the two single features and 
the dual feature rework state are given by:

$R w P_{2}=F\left(\left[L_{1}, U_{2}\right], \boldsymbol{\mu}, \boldsymbol{\Sigma}\right)-F(\boldsymbol{L}, \boldsymbol{\mu}, \boldsymbol{\Sigma})$

$+\sum_{i=2}^{\infty} R w P_{2_{i-1}}\left[1-F\left(U_{1}, \mu_{2,1}, \sigma_{1}\right)\right]+F(\boldsymbol{L}, \boldsymbol{\mu}, \boldsymbol{\Sigma})^{i-1}$

$\left[F\left(\left[L_{1}, U_{2}\right], \boldsymbol{\mu}, \boldsymbol{\Sigma}\right)-F(\boldsymbol{L}, \boldsymbol{\mu}, \boldsymbol{\Sigma})\right]$

$R w P_{3}=F\left(\left[U_{1}, L_{2}\right], \boldsymbol{\mu}, \boldsymbol{\Sigma}\right)-F(\boldsymbol{L}, \boldsymbol{\mu}, \boldsymbol{\Sigma})$

$+\sum_{i=2}^{\infty} R w P_{3_{i-1}}\left[1-F\left(U_{2}, \mu_{2,2}, \sigma_{2}\right)\right]+F(\boldsymbol{L}, \boldsymbol{\mu}, \boldsymbol{\Sigma})^{i-1}$

$\left[F\left(\left[U_{1}, L_{2}\right], \boldsymbol{\mu}, \boldsymbol{\Sigma}\right)-F(\boldsymbol{L}, \boldsymbol{\mu}, \boldsymbol{\Sigma})\right]$

$R w P_{4}=\sum_{i=1}^{\infty} F(\boldsymbol{L}, \boldsymbol{\mu}, \boldsymbol{\Sigma})^{i}$

The $F(\boldsymbol{L}, \boldsymbol{\mu}, \boldsymbol{\Sigma})^{i-1}$ term is a recursive term defining the rework entering the dual rework stage, equivalent to the last term in Equation 2. The scrap probabilities generated from the three rework states are given by Equation 21,

$$
\begin{aligned}
& \operatorname{SrP}_{2}=\left\{F\left(\left[L_{1}, U_{2}\right], \boldsymbol{\mu}, \boldsymbol{\Sigma}\right)-F(\boldsymbol{L}, \boldsymbol{\mu}, \boldsymbol{\Sigma})\right. \\
& +\sum_{i=2}^{\infty} R w P_{2_{i-1}}\left[1-F\left(U_{1}, \mu_{2,1}, \sigma_{1}\right)\right]+F(\boldsymbol{L}, \boldsymbol{\mu}, \boldsymbol{\Sigma})^{i-1} \\
& \left.\left[F\left(\left[L_{1}, U_{2}\right], \boldsymbol{\mu}, \boldsymbol{\Sigma}\right)-F(\boldsymbol{L}, \boldsymbol{\mu}, \boldsymbol{\Sigma})\right]\right\} F\left(L_{1}, \mu_{2,1}, \sigma_{1}\right)
\end{aligned}
$$

$$
\begin{aligned}
& \operatorname{Sr}_{3}=\left\{F\left(\left[U_{1}, L_{2}\right], \boldsymbol{\mu}, \boldsymbol{\Sigma}\right)-F(\boldsymbol{L}, \boldsymbol{\mu}, \boldsymbol{\Sigma})\right. \\
& +\sum_{i=2}^{\infty} R w P_{3_{i-1}}\left[1-F\left(U_{2}, \mu_{2,2}, \sigma_{2}\right)\right]+F(\boldsymbol{L}, \boldsymbol{\mu}, \boldsymbol{\Sigma})^{i-1} \\
& \left.\left[F\left(\left[U_{1}, L_{2}\right], \boldsymbol{\mu}, \boldsymbol{\Sigma}\right)-F(\boldsymbol{L}, \boldsymbol{\mu}, \boldsymbol{\Sigma})\right]\right\} F\left(L_{2}, \mu_{2,2}, \sigma_{2}\right),
\end{aligned}
$$

$S r P_{4}=S C_{4} \sum_{i=1}^{\infty}(1-F(\boldsymbol{U}, \boldsymbol{\mu}, \boldsymbol{\Sigma})) F(\boldsymbol{L}, \boldsymbol{\mu}, \boldsymbol{\Sigma})^{i}$.
To condense the notation let $T S r P=S_{r} P_{2}+\operatorname{SrP}_{3}+$ $\mathrm{SrP}_{4}$. Also the initial scrap cost is given from,

$\operatorname{Sr} P_{\mathrm{I}}=1-F(\boldsymbol{U}, \boldsymbol{\mu}, \boldsymbol{\Sigma})$,

The total profit for this two feature parallel processing example can be written as,

$$
\begin{aligned}
E(P R)_{2} & =S P\left[1-\left(T S r P\left(\boldsymbol{\mu}, \mu_{2,1}, \mu_{2,2}\right)+S r P_{\mathrm{I}}((\boldsymbol{\mu}))\right)\right] \\
& -\left[R c_{2} R w P_{2}\left(\boldsymbol{\mu}, \mu_{2,1}\right)+R c_{3} R w P_{3}\left(\boldsymbol{\mu}, \mu_{2,2}\right)\right. \\
& +R c_{4} R w P_{4}(\boldsymbol{\mu})+S c_{2} S r P_{2}\left(\boldsymbol{\mu}, \mu_{2,1}\right) \\
& \left.+S c_{3} S r P_{3}\left(\boldsymbol{\mu}, \mu_{2,2}\right)+S c_{4} S r P_{4}(\boldsymbol{\mu})\right]-P C .
\end{aligned}
$$

The single feature rework and scrap probabilities $\left(R w P_{2}\right.$, $R w P_{3}, S r P_{2}$ and $\operatorname{SrP}_{2}$ ) are functions of four means, $\boldsymbol{\mu}=\left[\mu_{1,1}, \mu_{1,2}\right]$ for dual feature rework and $\mu_{2,1}$ and $\mu_{2,2}$ for the two single feature reworks. This is because state 4 can feed state 2 , associated with $F\left(\left[U_{1}, L_{2}\right], \boldsymbol{\mu}, \boldsymbol{\Sigma}\right)$ and $F(\boldsymbol{L}, \boldsymbol{\mu}, \boldsymbol{\Sigma})$ and also state 3 associated with $F\left(\left[L_{1}, U_{2}\right], \boldsymbol{\mu}, \boldsymbol{\Sigma}\right)$ and $F(\boldsymbol{L}, \boldsymbol{\mu}, \boldsymbol{\Sigma})$. States 2 and 3 can feed themselves with components dependent on the probability of rework into states 2 and $3, F\left(U_{1}, \mu_{2,1}, \sigma_{1}\right)$ and $F\left(U_{2}, \mu_{2,2}, \sigma_{2}\right)$ respectively. Although $\mu_{1,1}$ and $\mu_{2,1}$ both apply to the feature $X_{1}$, the first mean only applies when the second feature is also processed along with the first feature prior to inspection. The second mean $\left(\mu_{2,1}\right)$ only applies when the first feature is processed and inspected independently from the second feature $\left(X_{2}\right)$. This also applies in a similar manner to the $X_{2}$ feature means. The reason for this distinction becomes apparent by considering what the optimal means would be for a single iteration of a dual feature example and a single feature example. Consider Figure 7 which shows the scatter of 2000 points drawn randomly from a joint normal distribution. The mean of the scattered points lies in the centre of the conformance region but due to the geometry of the scrap and rework regions, a greater proportion of these points lie in the scrap region since the scrap region is larger by $2 \mathrm{~L}_{1} \mathrm{~L}_{2}$ (difference between the scrap and rework areas). To ensure equal scrap and rework probability for the illustration in Figure $7, \mu_{x_{1}}=\mu_{x_{2}}=5.0617$. However, in a single feature case (Figure 1) there are equal probabilities of scrap and rework with the mean centred, $\mu=5$. This illustrates the optimal mean settings are different for dual and single feature manufacutring. To further complicate the balance between scrap and rework cost, the various rework regions (Figure 7) may have different costs associated with them and the cost of dual feature rework is likely to be the sum of the single feature rework costs. Therefore, to maximise the profit described by Equation 23, which 


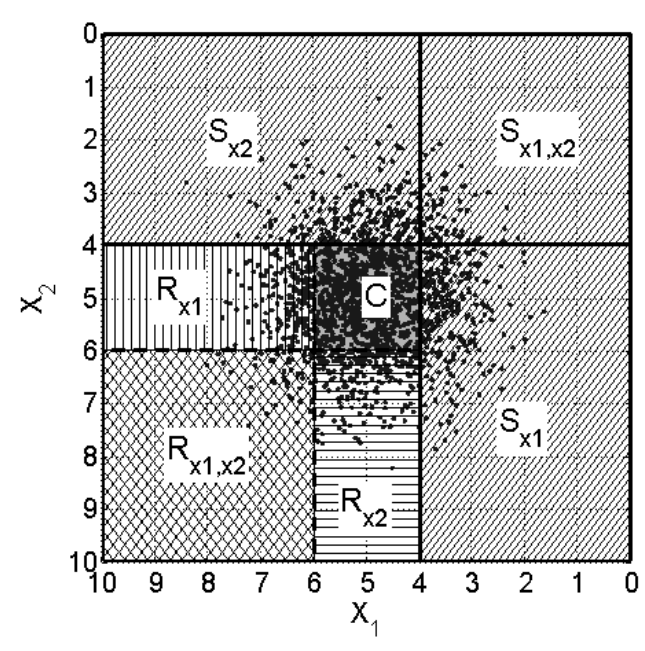

Fig. 7 Scatter plot with no correlation

has a mixture of dual feature and single feature rework, a total of four means must be adjusted such that,

$\hat{\boldsymbol{\mu}}_{\mathrm{opt}}=\max _{\hat{\boldsymbol{\mu}} \in \mathbb{R}}\left\{E(P R)_{2}\left(\mu_{1,1}, \mu_{1,2}, \mu_{2,1}, \mu_{2,2}\right)\right\}$.

The vector, $\hat{\boldsymbol{\mu}}_{\mathrm{opt}}$, is the four element vector containing the optimal means for the dual features as well as the single features. Note that the dual feature scrap and rework equations $\left(R w P_{4}\right.$ and $\left.\mathrm{SrP}_{4}\right)$ only involve dual feature probabilities and thus only the first two means of $\hat{\boldsymbol{\mu}}_{\mathrm{opt}}$ apply to these states. Clearly the exact values of the optimal means depend on the relative scrap and rework costs associated with the probabilities. A numerical example is shown in the following section to illustrate the impact of optimising the means for dual features separately from the single feature means.

\subsection{Dual Feature Numerical Example}

A dual feature optimal mean setting example was implemented to compare optimal mean setting using existing methodology (Khasawneh et al. [23]) and a new methodology. The existing methodology is referred to as Case I, where the means for each feature were kept constant for dual and single feature processing. The new methodology is referred to as Case II, where the means for dual feature processing were optimised independently of the means for single feature processing. Therefore, two means were optimised using the case I methodology and four means were optimised in the case II methodology. Two sets of cost values and statistical moments applicable to the dual feature optimal mean setting problem were available from Khasawneh et al. [23] and Peng and Khasawneh [25]. Different values have been used here to better graphically highlight

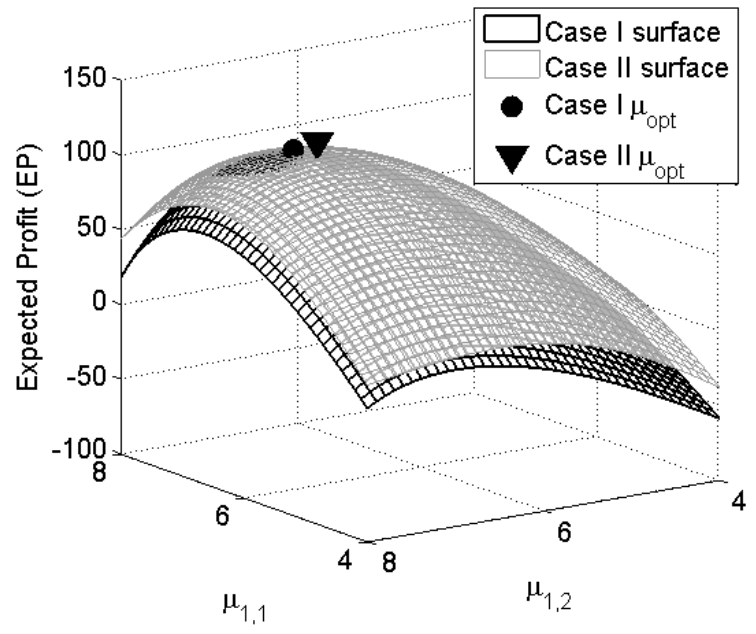

Fig. 8 Profit surfaces for Case I and Case II (optimisation of two and four means respectively)

the profit differences between the Case I and Case II methods. The cost values and statistical moments of the problem are shown in Table 2.

\begin{tabular}{|c|c|}
\hline Variable & Value \\
\hline $\mathbf{U}$ & {$[6 \quad 6]$} \\
\hline $\mathbf{L}$ & {$\left[\begin{array}{ll}4 & 4\end{array}\right]$} \\
\hline$R c$ & {$[252550]$} \\
\hline$S c$ & {$\left[100,100+\mathrm{Rw}_{2}, 100+\mathrm{Rw}_{3}, 100+\mathrm{Rw}_{4}\right]$} \\
\hline$S P$ & 500 \\
\hline$P C$ & 50 \\
\hline $\boldsymbol{\Sigma}$ & {$[2,0 ; 0,2]$} \\
\hline
\end{tabular}

Table 2 Dual feature numerical example input parameters

The expected profit given from Equation 23 was plotted for values of $\mu_{1,1}$ and $\mu_{1,2}$ in Figure 8. Case I represents the variability of expected profit for $\mu_{1,1}$ and $\mu_{1,2}$. The case II surface was generated by inputting a $\mu_{1,1}, \mu_{1,2}$ pair and resolving the $\mu_{2,1}$ and $\mu_{2,2}$ values by satisfying Equation 24 for the specified $\mu_{1,1}$ and $\mu_{1,2}$ inputs. The Matlab function 'fmincon' was used to implement this. The case II surface is higher at every point due to optimising the single feature rework means separately from dual feature processing. This also yielded a slightly different $\mu_{1,1}$ and $\mu_{1,2}$ optimum values, as there was no compromise between dual feature and single feature cost. The dual feature means were lower than the single feature means, primarily due to the $R_{X_{1}, X_{2}}$ rectangle in (Figure 10). Components falling into this region $\left(R_{X_{1}, X_{2}}\right.$ rectangle) experienced double the single feature rework cost as well as the increased probability of further rework. This double feature rework state does not exist for a single feature, which allows the single feature means to be biased to a greater extent 


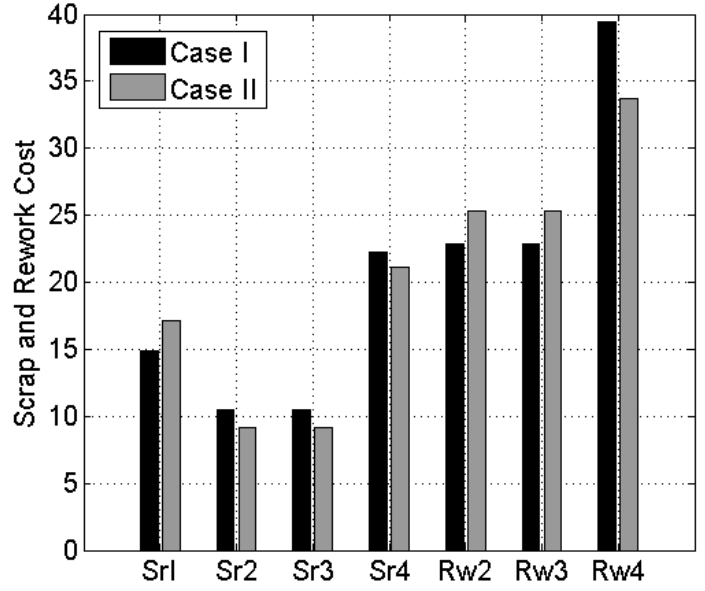

Fig. 9 Scrap and rework costs from the initial and rework states

towards rework than the double feature case, without incurring a cost penalty. The profits and optimal mean settings are displayed in Table 3 which correspond to the markers on Figure 8.

\begin{tabular}{|c|c|}
\hline & Value \\
\hline Case I Profit & 103.26 \\
\hline Case II Profit & 105.29 \\
\hline Case I Production Cost & 143.10 \\
\hline Case II Production Cost & 140.84 \\
\hline Case I means $\left(\mu_{1,1}^{\mathrm{I}}, \mu_{1,2}^{\mathrm{I}}\right)$ & $6.85,6.85$ \\
\hline Case II means $\left(\mu_{1,1}^{\mathrm{II}}, \mu_{1,2}^{\mathrm{II}}, \mu_{2,1}^{\mathrm{II}}, \mu_{2,2}^{\mathrm{II}}\right)$ & $6.69,6.69,7.11,7.11$ \\
\hline Case I Final Conformance Prob. & 0.5927 \\
\hline Case II Final Conformance Prob. & 0.5922 \\
\hline Case I Final Scrap Prob. & 0.4073 \\
\hline Case II Final Scrap Prob. & 0.4078 \\
\hline
\end{tabular}

Table 3 Optimisation results

The bar plot in Figure 9 shows the rework and scrap costs from the initial and rework states, illustrated on Figure 5. The initial scrap cost was less for case I compared to case II $\left(\mathrm{Sr}_{\mathrm{I}}\right.$ bar in Figure 9$\left.)\right)$ as $\mu_{1,1}^{\mathrm{I}}$ and $\mu_{1,2}^{\mathrm{I}}$ were more rework biased than $\mu_{1,1}^{\mathrm{II}}$ and $\mu_{1,2}^{\mathrm{II}}$. Consequentially, the dual feature rework cost $\mathrm{Rw}_{4}$ was comparatively high due to this rework bias. The last two Case II means, $\mu_{2,1}^{\mathrm{II}}$ and $\mu_{2,2}^{\mathrm{II}}$, which applied to single feature rework, were higher than $\mu_{1,1}^{\mathrm{I}}$ and $\mu_{1,2}^{\mathrm{I}}$, generating less $\mathrm{Sr}_{2}$ and $\mathrm{Sr}_{3}$ scrap from Case II but more $\mathrm{Rw}_{2}$ and $\mathrm{Rw}_{3}$ rework. The greater cost of dual feature scrap, $\mathrm{Sr}_{4}$ for case I, was due to the increased proportion of components in the $\mathrm{Rw}_{4}$ state. Overall, the reduced scrap and dual feature rework costs from the case II led to a $1.92 \%$ increase in profit over case I. It is also important to note from Table 3 , that the number of items eventually conforming was slightly higher in case

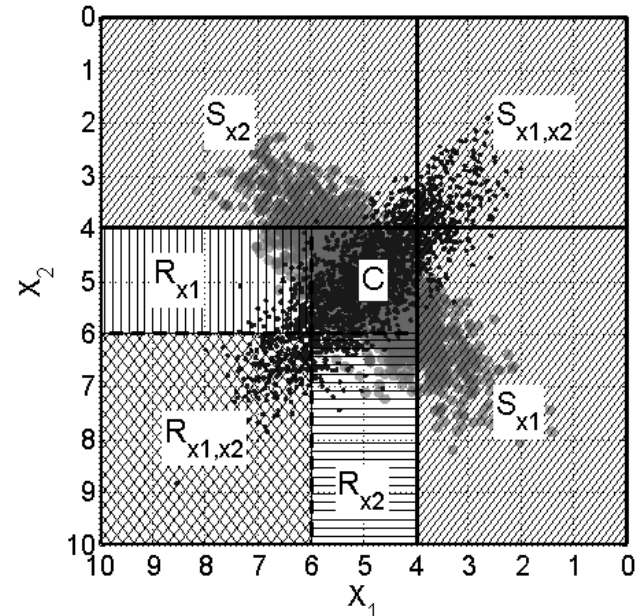

Fig. 10 Scatter plot with correlation $(\rho=-0.8$ and $\rho=0.8)$

I and hence more components could be sold, however this was offset by the reduction in scrap and rework cost achieved in case II.

\subsection{Influence of Correlation}

Correlation alters the probability of components falling into single and dual feature rework states, which in turn may influence the optimal means. Figure 10 indicates the scatter of points with correlation, $\rho=0.8$ and $\rho=$ -0.8 where the correlation matrix is

$$
\boldsymbol{\Sigma}=\left[\begin{array}{cc}
\sigma_{1} & \rho \sigma_{1} \sigma_{2} \\
\rho \sigma_{1} \sigma_{2} & \sigma_{2}
\end{array}\right]
$$

The darker points correspond to the $\rho=0.8$ value, while the lighter points correspond to $\rho=-0.8$. Table 4 indicates the differences between the number of points falling in the various regions after one processing operation (defined in Figures 6 and 7 ) compared to the uncorrelated example, where $\rho=0$. Both positive and negative correlation almost halved the probability of points falling in the single feature rework regions compared to no correlation. Conformance was increased in both cases. The changes in scrap and dual feature rework depended on the sign of the correlation parameter $\rho$. Positive correlation almost quadrupled the probability of dual feature rework and reduced the probability of scrap by around a quarter. Negative correlation reduced the probability of dual feature rework by a factor of over 500 and slightly increased the probability of scrap. This is clear from the orientations of the point clusters in Figure 10.

The effect of correlation on the optimal means and profits are tabulated in Table 5. For $\rho=0.8$, profits 


\begin{tabular}{|c|c|c|}
\hline Region & Ratio, $\rho=0.8$ & Ratio, $\rho=-0.8$ \\
\hline $\mathrm{R}_{\mathrm{x} 1}$ & 0.5632 & 0.5623 \\
\hline $\mathrm{R}_{\mathrm{x} 2}$ & 0.5632 & 0.5623 \\
\hline $\mathrm{R}_{\mathrm{x} 1, \mathrm{x} 1}$ & 3.8730 & 0.0022 \\
\hline $\mathrm{C}$ & 1.203 & 1.203 \\
\hline $\mathrm{S}$ & 0.7515 & 1.0880 \\
\hline
\end{tabular}

Table 4 Impact of correlation on the probability of components falling into rework, scrap and conformance

were greater for both Case I and II over the uncorrelated example due to a reduced scrap and rework cost and higher overall conformance. The profit increase for $\rho=-0.8$ for cases I and II was solely due to the reduction in production cost (scrap and rework cost), the final conformance was slightly lower than the uncorrelated example (Table 3).

The case I means for $\rho=0.8$ are lower than the case I means with no correlation $(\rho=0)$. The means were required to be lower to reduce the proportion of components falling into the $R_{X_{1}, X_{2}}$ region given correlation increased the probability of $R_{X_{1}, X_{2}}$ rework. The $\mu_{1,1}^{\mathrm{II}}$ and $\mu_{1,2}^{\mathrm{II}}$ means for $\rho=0.8$ are lower than the $\mu_{1,1}^{\mathrm{II}}$ and $\mu_{1,2}^{\mathrm{II}}$ means for $\rho=0$, for the same reason. They were also lower than the $\mu_{1,1}^{\mathrm{I}}$ and $\mu_{1,2}^{\mathrm{I}}$ means (for $\rho=0.8$ ) as they were optimised separately to the single feature means. Note, the $\mu_{2,1}^{\mathrm{II}}$ and $\mu_{2,2}^{\mathrm{II}}$ means are very similar to the uncorrelated case $(\rho=0)$. Recall, these means only applied to single feature rework and were unaffected by correlation. The reason they were not exactly the same is due to the dual feature processing. With correlation, the probability of producing single feature rework was reduced, while the probability of producing dual feature rework was increased. Equation 20 indicates this reduced the single feature rework probabilities faster than the increased probability of dual feature rework, thus the rework cost was reduced allowing slightly more rework biased means for the same cost.

The case I means for $\rho=-0.8$ were also lower than in the uncorrelated case. To reduce cost, the extremities of the negatively correlated scatter region in Figure 10 moved to reduce the probability of scrap but not so far to make rework, specifically $R_{x 1} R_{x 2}$ too significant. This was achieved by shifting the mean of the distribution towards to $(0,0)$ compared to the uncorrelated case $(\rho=0)$, but to a lesser extent than in the positive correlated case $(\rho=0.8)$. The $\mu_{1,1}^{\mathrm{II}}$ and $\mu_{1,2}^{\mathrm{II}}$ means of case II, where $\rho=-0.8$, are also lower than the uncorrelated case for the same reason and again lower than $\mu_{1,1}^{\mathrm{I}}$ and $\mu_{1,2}^{\mathrm{I}}$ due to the differences between the case I and case II methodologies (as explained in Section 4.1). The $\mu_{2,1}^{\text {II }}$ and $\mu_{2,2}^{\mathrm{II}}$ means from Case II were very similar to the positive correlated case and the uncorrelated case due to the fact they only applied to single feature rework and therefore were not directly affected by correlation. However, there were slight differences in the third decimal point due to different probabilities of single and dual feature rework from dual feature processing, as explained in the previous paragraph.

\begin{tabular}{|c|c|c|}
\hline$\rho$ & Cases & Value \\
\hline 0.8 & Case I Profit & 117.43 \\
\hline 0.8 & Case II Profit & 120.96 \\
\hline 0.8 & Case I Production Cost & 139.65 \\
\hline 0.8 & Case II Production Cost & 136.75 \\
\hline 0.8 & Case I Final Conformance & 0.6142 \\
\hline 0.8 & Case II Final Conformance & 0.6154 \\
\hline 0.8 & Case I means & $6.63,6.23$ \\
\hline 0.8 & Case II means & $6.45,6.45,7.12,7.12$ \\
\hline-0.8 & Case I Profit & 109.72 \\
\hline-0.8 & Case II Profit & 114.25 \\
\hline-0.8 & Case I Production Cost & 132.86 \\
\hline-0.8 & Case II Production Cost & 125.17 \\
\hline-0.8 & Case I Final Conformance & 0.5852 \\
\hline-0.8 & Case II Final Conformance & 0.5788 \\
\hline-0.8 & Case I means & $6.79,6.79$ \\
\hline-0.8 & Case II means & $6.45,6.45,7.11,7.11$ \\
\hline
\end{tabular}

Table 5 Optimisation results for correlated features

The sensitivity of profit to correlation is plotted in Figure 11 for both cases. The difference between the two cases is shown by the grey dotted line and corresponds to the scale on right hand y-axis. In general, the greater the degree of positive or negative correlation the higher the profit with a minimum profit existing at $\rho \approx 0.3$. The actual minimum profit for a given correlation depended on the geometry of the scrap and rework regions and relative standard deviations and tolerance bounds of each feature. As $\rho \rightarrow 1$ the difference between the two and four mean case diminished as all components designated rework lay in the dual feature rework region. Thus, the benefit of separately optimising the single feature rework means was lost as there was little or no single feature rework. This is evident by considering Figure 10, the darker points would converge on a single diagonal as $\rho \rightarrow 1$. The same converging type effect occurs for $\rho \rightarrow-1$, although the line orientation is changed by 90 degrees. However, as can be seen from Figure 10 (the lighter) points will remain in the single feature rework regions as $\rho \rightarrow-1$. It is also likely dual feature rework will exist (depending on the geometry of the scrap and rework regions and the standard deviation of the feature variation) thus, there is still a benefit to optimising dual and single feature means separately. This led to a profit difference between case I and II for negative correlation. 


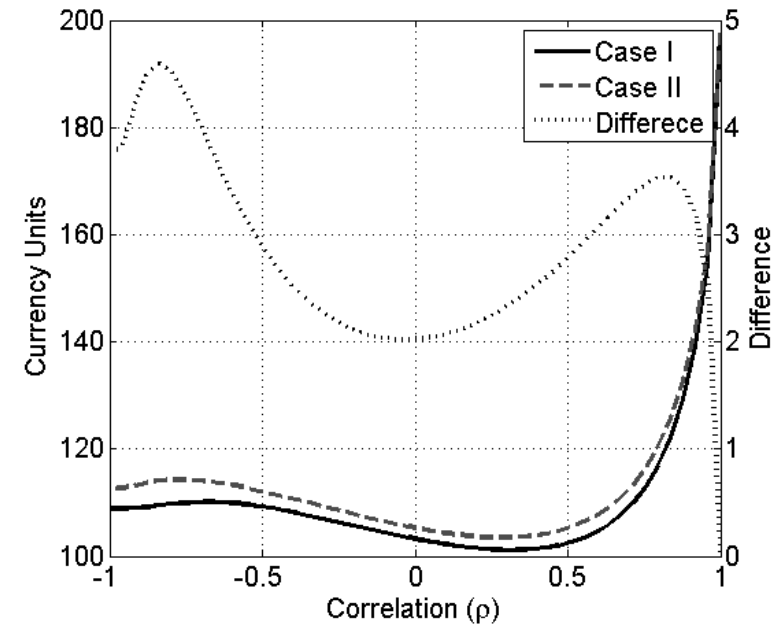

Fig. 11 Profit vs. correlation

\section{Conclusion and Future Work}

There were three primary contributions made by this article. Firstly, a single feature optimal mean setting problem was examined from first principles to investigate the impact on expected profit with multiple means for each rework iteration. It was proven that expected profit was maximised when the same optimal mean was applied for each iteration. Thus, the application of Markovian modelling to find the optimal mean setting (introduced by Bowling et al. [22]) was justified (Markov modelling implicitly assumes the same mean is applied to all rework iterations). Secondly, the maximum obtained profit for dual feature optimal mean setting was increased by $1.92 \%$ in comparison to the results attained by the method presented in the leading articles in the field. This was achieved by optimising the dual and single feature means separately, following from the speculation in the single feature rework problem, that a separate mean could be assigned to each rework iteration. Finally, the effect of correlation on profit was considered. Correlation was generally found to have a positive effect on the expected profit, where again a greater profit was attainable by optimising the dual feature means separately from the single feature means, for a dual feature example.

A natural extension to this work would be to investigate the effect of more than two features being processed prior to inspection. For example, applying this methodology to processing three features prior to inspection would involve optimising the means for triple feature processing, dual feature processing and single feature processing, nine individual means. This will yield greater differences in profit compared to using the case I methodology, (solely optimising the three feature means).
The effect of optimal mean setting on the final geometry of the features is also worth considering. The geometry will be represented by a truncated Gaussian mixture model where two or more features are processed prior to inspection.

Acknowledgements This work was conducted through a Rolls-Royce plc. led research programme SILOET (Strategic Investment in Low Carbon Engine Technology). Funding was provided by Rolls-Royce, TSB (Technology Strategy Board) and the EPSRC (Engineering and Physical Sciences Research Council).

\section{References}

1. C Springer. A Method for Determining the Most Economic Position of a Process Mean. Industrial Quality Control, 8(1):36-39, 1951.

2. W.G. Hunter and C.P Kartha. Determining the Most Profitable Value for a Production Process. Journal of Quality Technology, 9:176-181, 1977.

3. L.S. Nelson. Best Target Value for a Production Process. Journal of Quality Technolgy, 10(2):8889, 1978.

4. Carlsson. Determining the most profitable process level for a process under different sales conditions. Journal of Quality Technolgy, 16(1):44-49, 1984.

5. S. Bisgaard, W Hunter, and L. Pallensen. Economic Selection of Quality of Manufactured Product. Technometrics, 26(1):9-18, 1984.

6. D.C. Bettes. Finding an Optimum Target Value in Relation to a Fixed Lower Limit and an Arbitrary Upper Limit. Journal of the Royal Statistical Society. Series C (Applied Statistics), 11(3):202-210, 1962.

7. Y.D. Golhar and S.M. Pollock. Determination of Optimal process mean and the upper limit for a canning problem.pdf. Journal of Quality Technology, 20:188-192, 1988.

8. R. Schmidt and P. Pfeifer. Economic Selection of the Mean and Upper Limit for a Canning Problem with Limited Capacity. Journal of Quality Technology, 23(4):312-317, 1991.

9. W. Liu and M. Raghavachari. The Target Mean Problem for an Arbitrary Quality Characteristic Distribution. International Journal of Production Research, 35(6):1713-1728, June 1997. ISSN 0020-7543. doi: 10.1080/002075497195227. URL http://www .tandfonline.com/doi/abs/10 . 1080/0020754971

10. D Wen and A.E. Mergen. Running a process with poor capability. Quality Engineering, 11(4):505-509, July 1999. ISSN 0898- 
2112. doi: 10.1080/08982119908919270. URL 21. DY. Golhar. Determination of the best means conhttp://www.tandfonline.com/doi/abs/10.1080/089821thes8)r9870anning problem. Journal of Quality

11. C.H. Chen, C.Y. Chou, and K.W. Huang. Determining the Optimum Process Mean Under Quality Loss Function. The International Journal of Advanced Manufacturing Technology, 20:598-602, 2002.

12. Jirarat Teeravaraprug and Byung Rae Cho. Designing the optimal process target levels for multiple quality characteristics. International Journal of Production Research, 40(1):37-54, January 2002. ISSN 00207543. doi: 10.1080/00207540110073046. URL http://www.tandfonline.com/doi/abs/10.1080/

13. L.L. Ho and Roberto C. Quinino. Optimum Mean Location in a Poor-Capability Process. Quality Engineering, 16(2):257-263, January 2003. ISSN 0898-2112. doi: 10.1081/QEN-120024014. URL http://www. tandfonline.com/doi/abs/10.1081/

14. C.H. Chen and C.Y. Chou. Determining the Optimum Process Mean Under the Bivariate Quality Characteristics. The International Journal of Advanced Manufacturing Technology, 21:313-316, 2003.

15. Genichi Taguchi. Introduction to quality engineering. Asian Productivity Organization, New York, 1986.

16. E.A. Elsayed and A. Chen. Optimal levels of Process Parameters for Products with Multiple Characteristics. International Jounral of Production Research, 5(31):1117-1132, 1993.

17. D. Drain and A.M. Gough. Applications of the Upside-Down Normal Loss Function. IEEE Transaction on Semiconductor Manufacturing, 9(1):143$145,1996$.

18. K.C. Kapur and B.R. Cho. Economic design of the specification region for multiple quality characteristics. IIE Transactions, 28(3):237-248, March $1996 . \quad$ ISSN 0740817X. doi: $10.1080 / 07408179608966270$. URL http://www.tandfonline.com/doi/abs/10.1080/0740

19. W.M. Chan and R.N. Ibrahim. Evaluating the quality level of a product with multiple quality characterisitcs. The International Journal of Advanced Manufacturing Technology, 24(9-10):738-742, April 2004. ISSN 0268-3768. doi: 10.1007/s00170-003-1751-6. URL http://link.springer.com/10.1007/s00170-003

20. K.S. Al-Sultan and M.F.S. Pulak. Optimum target values for two machines in series with $100 \%$ inspection. European Journal of Operational Research, 120:181-189, 2000.
Technology, 19:82-84, 1987.

22. S.R. Bowling, M.T. Khasawneh, S. Kaewkuekool, and B.R. Cho. A Markovian approach to determining optimum process target levels for a multistage serial production system. European Journal of Operational Research, 159(3):636-650, December 2004. ISSN 03772217. doi: 10.1016/S03772217(03)00429-6.

23. Mohammad T. Khasawneh, Shannon R. Bowling, and Byung Rae Cho. A Markovian approach to determining process means with dual quality char-

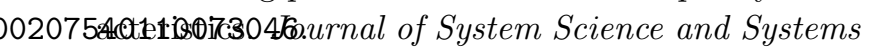
Engineering, 17(1):66-85, March 2008. ISSN 10043756. doi: 10.1007/s11518-008-5064-z.

24. Shokri Z. Selim and Walid K. Al-Zubi. Optimal means for continuous processes in series.

/QEN-12DQ2APAfan Journal of Operational Research, 210 (3):618-623, May 2011. ISSN 03772217. doi: 10.1016/j.ejor.2010.10.013.

25. Chien-Yi Peng and Mohammad T. Khasawneh. A Markovian approach to determining optimum process means with inspection sampling plan in serial production systems. International Journal of Advanced Manufacutring Technology, 72(9-12): 1299-1323, March 2014. ISSN 0268-3768. doi: 10.1007/s00170-014-5741-7.

26. Paul L. Goethals and Byung Rae Cho. Solving the optimal process target problem using response surface designs in heteroscedastic conditions. International Journal of Production Research, 49(12):3455-3478, June 2011. ISSN 00207543. doi: $10.1080 / 00207543.2010 .484556$. URL http://www.tandfonline.com/doi/abs/10.1080/00207543.2C

27. P.L. Goethals and B.R. Cho. Designing the optimal process mean vector for mixed multiple quality characteristics. IIE Transactions, 44 (11):1002-1021, November 2012. ISSN 0740817X. doi: 10.1080/0740817X.2012.655061. URL

74081706p896616027 Ot andf online . com/doi/abs/10 .1080/0740817X. $2 \mathrm{C}$

28. Gregory L. Boylan and Byung Rae Cho. Solving the Multidisciplinary Robust Parameter Design Problem for Mixed Type Quality Characteristics under Asymmetric Conditions. Quality and Reliability Engineering International, 30(5):681-695, July 2014. ISSN 07488017. doi: $10.1002 /$ qre.1520. URL

1751-16ttp://doi . wiley.com/10.1002/qre. 1520.

29. A. Azzalini. A Class of Distributions which includes Normal Ones. Scandinavian Journal of Statistics, 12:171-178, 1985.

30. Roger Nelsen. An Introduction to Copulas. Springer, New York, 2nd ed. edition, 2006. ISBN 
10: $0-387-28659-4$.

\section{Appendix}

\subsection{The Nature of the Stationary Points}

Theorem 1 was proven and total profit is maximised when the same optimal mean is applied over all rework iterations such that, $\mu=\mu_{\mathrm{opt}}$. Thus the expression for total profit, Equation 2, can be formulated as a geometric series and written,

$$
\begin{aligned}
T P & =S P\left(\frac{F(U, \mu, \sigma)-F(L, \mu, \sigma)}{1-[1-F(U, \mu, \sigma)]}\right) \\
& -P C-S c\left(\frac{F(L, \mu, \sigma)}{1-[1-F(U, \mu, \sigma)]}\right) \\
& -R c\left(\frac{1}{1-[1-F(U, \mu, \sigma)]}-1\right) .
\end{aligned}
$$

The nature of the stationary point which maximised $T P$ is given by,

$$
\begin{aligned}
\frac{\mathrm{d}^{2} T P}{\mathrm{~d} \mu^{2}} & =\frac{\sqrt{2}}{\sqrt{\pi} \sigma^{3} G(\mu)}((L-\mu) A(\mu) \xi(v) \alpha \\
& -(U-\mu) B(\mu) \xi(\varphi) \alpha-(2 R c+\alpha)(U-\mu) B(\mu) \\
& +(L-\mu) A(\mu))+\frac{4 B(\mu)}{\pi \sigma^{2} G(\mu)^{3}}(A(\mu) \xi(v) \alpha \\
& -B(\mu) \xi(\varphi) \alpha+(2 R c+\alpha) B(\mu)-A(\mu) \alpha) .
\end{aligned}
$$

The functions $A, B$ and $G$ are given by

$$
\begin{gathered}
A(\mu)=\exp \left(-\frac{(L-\mu)^{2}}{2 \sigma^{2}}\right) \\
B(\mu)=\exp \left(-\frac{(U-\mu)^{2}}{2 \sigma^{2}}\right) \\
G(\mu)=1+\frac{2}{\sqrt{\pi}} \int_{0}^{\frac{(U-\mu) \sqrt{2}}{2 \sigma^{2}}} e^{-t} \mathrm{~d} t
\end{gathered}
$$

The stationary point of Equation 25 is only a maximum when $\mathrm{d}^{2} T P / \mathrm{d} \mu^{2}<0$. This condition is generally satisfied for $S P>S c>R c$, which ensures that the optimal mean lies to the right of the nominal mean, $\mu_{\text {nom }}$. While $\mu_{\text {opt }}<U S L$ there are only two positive contributing terms in Equation 25, the third and eighth terms, $-(U-\mu) B(\mu) \xi(\varphi) \alpha$ and $-B(\mu) \xi(\varphi) \alpha$, because $\xi(\varphi)$ may be negative as indicated by Figure 12 . However, the absolute value of the fourth and ninth terms

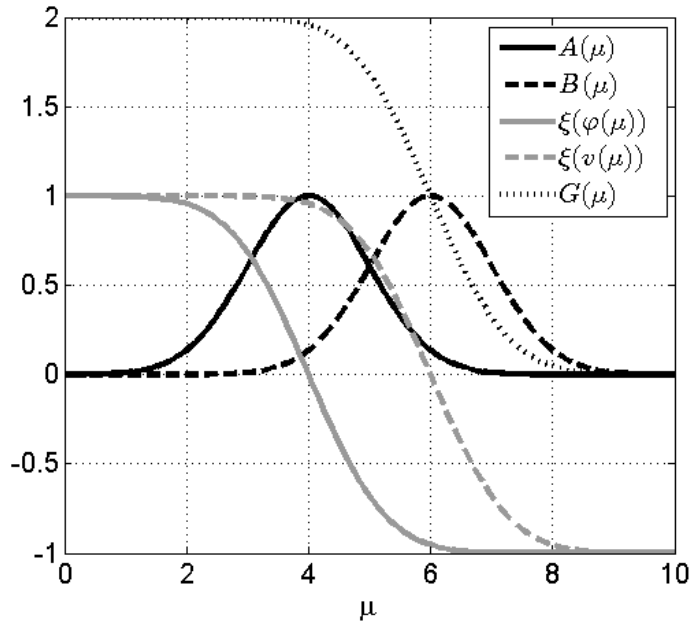

Fig. 12 Illustration of the $A, B, \xi(\varphi), \xi(v), G(\mu)$ functions for $L=4, U=6$ and $\sigma=1$

is always greater than the third and eighth respectively such that,

$$
|(2 R c+\alpha)(U-\mu) B(\mu) \xi(\varphi)|>|(U-\mu) B(\mu) \xi(\varphi) \alpha|
$$

and

$$
|(2 R c+\alpha) B(\mu)|>|B(\mu) \xi(\varphi) \alpha|
$$

making the sum negative, and thus Equation 26 remains negative.

While $\mu_{\mathrm{opt}}>U S L$ the second and seventh terms from Equation 26 make a positive contribution due to $\xi(v)$ becoming negative, as illustrated on Figure 12 . Never-the-less the absolute value of the fifth term and tenth terms are greater than the second and seventh terms respectively such that,

$$
|(L-\mu) A(\mu)| \geq|(L-\mu) A(\mu) \xi(v)|
$$

and

$$
|A(\mu) \alpha| \geq|A(\mu) \xi(v) \alpha|
$$

which again ensures Equation 26 is negative confirming the stationary point is a maximum $\forall \mu \in \mathbb{R}$ where $\mu_{\text {opt }}>\mu_{\text {nom }}$. It is worth clarifying in practical cases $\mu_{\text {opt }}>\mu_{\text {nom }}$ since the selling price must be greater than the scrap cost which in general is greater than the rework cost $(S P>S c>R c)$. 